\title{
An Improved Clusterion-Photoelectron Coincidence Technique for the Investigation of the Ionisation Dynamics of Clusters
}

\author{
L. Cordis, G. Ganteför, J. Heflich, and A. Ding \\ Hahn-Meitner-Institut für Kernforschung, Bereich Strahlenchemie, Berlin, Germany
}

\begin{abstract}
The introduction of photoion-photoelectron coincidence techniques has made it possible to investigate photoionisation properties of heavy clusters, which are not accessible by conventional mass spectrometry. This technique has been further developed in combination with a zero-volt electron energy analyser and greatly improved in performance.

The method has been applied to the investigation of different homogeneous and heterogeneous clusters. This type of cluster experiment requires both a very high resolution and a large dynamic range in order to identify also clusters present in low abundance.

As an example, a series of coincidence mass spectra of Xe clusters has been recorded at different wavelengths. Below a photon energy of $11.1 \mathrm{eV}$, the range of observable clusters shifts to higher cluster sizes with decreasing energy. Appearance potentials and the binding energy of different cluster ions were obtained. Intensity fluctuations, already observed in spectra with electron bombardment ionisation (magic numbers), have also been detected in the coincidence spectra and become most pronounced near the ionisation threshold. This indicates that these fluctuations are caused by the size-dependent stability of the ionic and not the neutral cluster. Furthermore, the threshold size does not change linearly with cluster size. The binding energy per particle seems to change drastically around $n=13$ which indicates the existence of a shell structure in the cluster ion.
\end{abstract}

PACS: $33.65 ; 33.80 \mathrm{E} ; 36.40$

\section{Introduction}

Interest in the properties of neutral and ionic clusters has grown rapidly in recent times. Besides investigating intermolecular forces [1] and chemical dynamics [2], it is particularly interesting to study the transition between the gas phase and the condensed state of matter. Recently, the study of surface phenomena like chemisorption [3] in isolated systems and surface reactivity [4] of clusters has become feasible through the development of many new experimental techniques for the investigation of free cluster beams of very different composition.

A major obstacle in the understanding of cluster properties is the lack of a genuinely size-sensitive detection procedure. Methods such as spectroscopy or electron scattering fail to provide direct insight into the size distribution of clusters while mass spectroscopy requires ionisation of the cluster.

Extensive fragmentation occurs during the ionisation process due to the energy released as the cluster changes from the neutral to the ionic equilibrium configuration. A prerequisite for the understanding of the fragmentation process is knowing the amount of energy dissipated in the cluster. For this reason a detailed energy balance of the system before and after ionisation is needed. For photoionisation, the energy dissipated in the system is the energy of the photon. It is partly released as internal energy in the cluster with the remaining energy appearing as kinetic energy of the ejected electron. In order to collect more exact information, it is necessary to measure the kinetic energy of the ejected electron.

Electron bombardment ionisation is even more 
complex, as the energy of the system is distributed between 3 particles - as internal energy of the cluster and as kinetic energy of both electrons.

\section{Experimental Method}

On the basis of previously developed PIPECO and TEPICO $[5,6]$ methods we have devised an improved method for investigating the energy released into a cluster after photoionisation: pairs of electrons and cluster ions are produced by irradiating a molecular beam containing a small percentage $(1 \%$ to $2 \%)$ of clusters with monochromatic VUV light from the Berlin Synchrotron Source BESSY. A weak continuous electric field separates the ion-electron pairs produced by photoionisation. Electrons below a certain energy determined by the electric field are extracted into a drift region consisting of an axial cylinder with a channeltron multiplier attached to an aperture on the side of the cylinder (Fig. 1). The field penetration caused by the high electric potential of the multiplier serves to guide the electrons towards the multiplier. The majority of the electrons with energies higher than the threshold is emitted perpendicular to the axis and lost. Only those electrons emitted in the direction of the axis will be collected and eventually limit the energy resolution.

After amplification and pulse shaping, the multiplier signal is used to start a time-to-amplitude converter. It also triggers a pulse generator which applies a high voltage pulse to a repeller electrode inside the primary ionisation chamber, thus accelerating the ion towards the detector (c.f. Fig. 1). The ion then undergoes continuous acceleration in a section of constant electric field and passes through a field-free drift region before reaching the ion detector. The pulse height of the repeller pulse, the length of the acceleration region, the length of the drift region and the final acceleration potential were chosen so as to achieve spatial focussing, thus optimising the mass resolution. By using a low extraction field for the electron and subsequently a pulsed accelerating field for the ion, both the energy resolution of the electron and the time resolution of the ion can be optimised independently. The coincidence methods used without the extraction pulse yielded high energy resolution of the ejected electrons but had an inferior time resolution since the ions remained for a longer time in the low extraction field. The reason for this is that the time-of-flight depends on the location where the ions are produced and shows large variations due to the finite diameter of the photon beam.

Furthermore, the coincidence method shares all the advantages of the time-of-flight techniques without requiring installation of pulsed ion sources. It is therefore possible to use it in connection with continuous or quasi continuous light sources. This is of particular interest for the use of synchrotron radiation for time-of-flight photoionisation mass spectrometry, as the pulse structure of a synchrotron is usually too fast to be used with conventional time-offlight techniques. A disadvantage of the coincidence technique is, however, that no other electron ion pair may be generated during the travelling time of the ion as the technique relies on correlation between the electron and ion formed from the same particle. This limits the maximum achievable counting rate to approximately one third of the inverse maximum flight time. Higher rates will result in additional statistical coincidences, whose number increases quadratically with the average counting rates.

Using high acceleration voltages $(1 \mathrm{kV})$ and short drift tubes $(10 \mathrm{~cm})$, maximum rates of approximately $10 \mathrm{kHz}$ are feasible. In the previously used setup only 1 to $2 \mathrm{kHz}$ had been obtained.

The coincidence method combines the following advantages of conventional time-of-flight mass spectrometry and photoelectron spectroscopy:

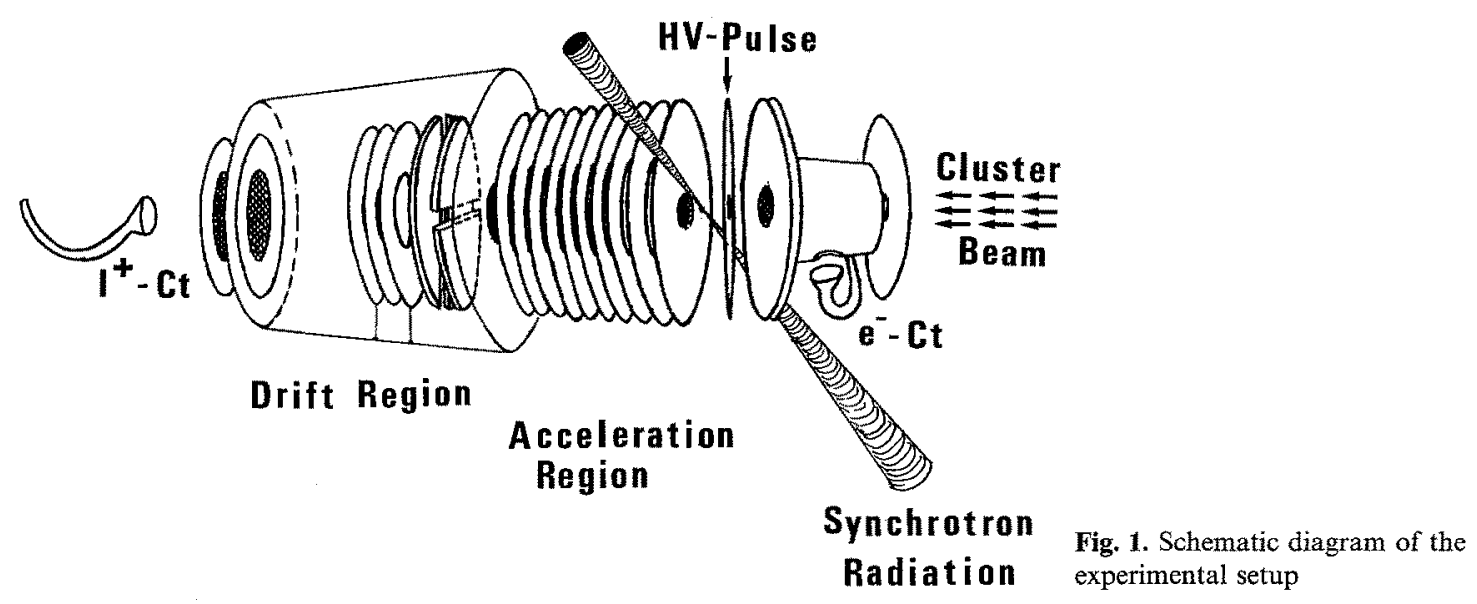




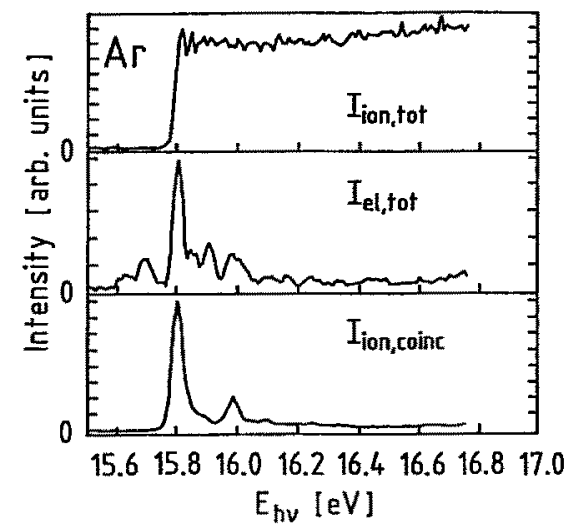

Fig. 2. Photoionisation efficiency curve (top), photoelectron spectrum (middle) and photoion-photoelectron coinicidence spectrum (bottom) of a supersonic argon beam

1) It is possible to record mass spectra over an exceptionally large mass range. This is a particular advantage in conjunction with cluster experiments as large clusters cannot be accessed using conventional quadrupole or magnetic mass spectrometers.

2) By using electronic gates, it is possible to scan several masses simultaneously as a function of photon energy. The number of channels is limited only by the electronic equipment available. This multiplex gain constitutes a great advantage compared with the standard techniques and makes the method one of the most sensitive ones. The absence of narrow slits increases the sensitivity further.

3) It is possible to perform mass selective electron spectroscopy. This is particularly useful when dealing with systems containing a large number of constituents of different masses as in cluster experiments. Figure 2 compares the different methods of photoionisation mass spectrometry, conventional electron spectroscopy and the coincidence method applied to the analysis of supersonic expansion of argon gas. The supersonic argon beam contains a small fraction of dimers and larger clusters. In spite of the photoelectron spectrum (middle) exhibiting many more details than the standard photoionisation efficiency curve (top), interpretation is very difficult if more than one mass contributes to the spectrum. The bottom curve shows the photoion-photoelectron coincidence spectrum for a selected mass range (argon monomer). The direct ionisation channels leading to the ${ }^{2} P_{3 / 2}$ and the ${ }^{2} P_{1 / 2}$ ionic states of the argon ion are clearly visible.

Similar spectra have been taken for the masses of the higher multimers. An example of a $\mathrm{Kr}_{2}^{+}$coincidence spectrum is shown in Fig. 3 together with the positions of the molecular Rydberg states of the excited krypton dimer. Compared with the results of $\mathrm{Ng}$ et al. [6], the spectrum shows a higher degree of

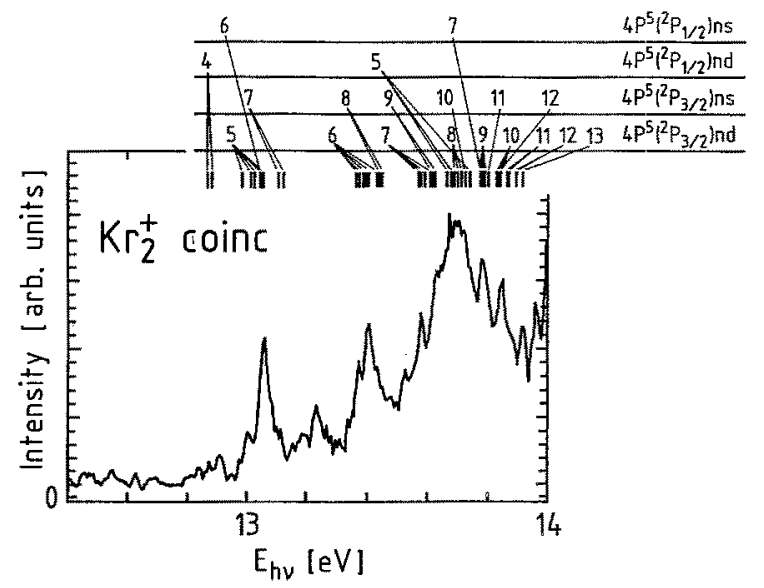

Fig. 3. Photoion-photoelectron coincidence spectrum of the krypton dimer ion

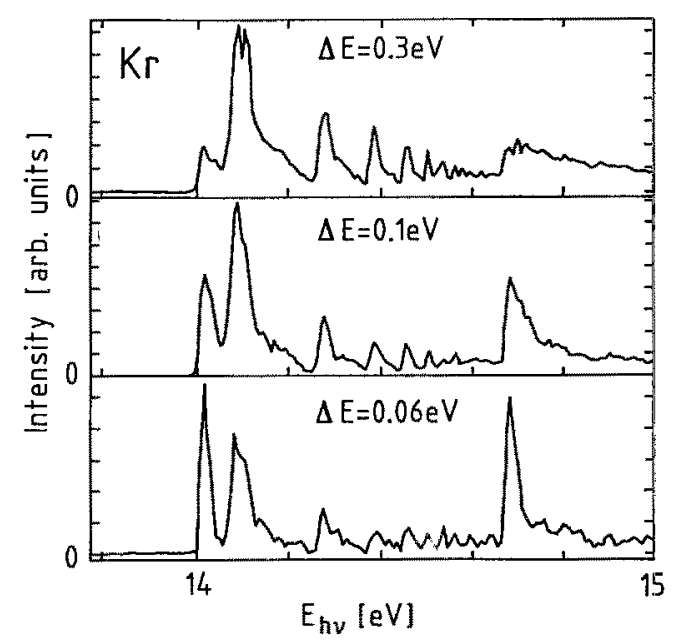

Fig. 4. The effect of the resolution of the zero-volt electron energy analyser on the spectrum of krypton atoms

modulation and different relative peak heights. This is not surprising as the spectrum in Fig. 3 displays the contribution of only those ions generated together with threshold electrons.

The resolution of the zero-volt energy analyser can be changed by variation of the extraction field. Figure 4 shows electron-ion coincidence spectra of $\mathrm{Kr}$ atoms using different resolution of the electron analyser. The direct ionisation of $\mathrm{Kr}$ into $\mathrm{Kr}^{+}{ }^{2} P_{3 / 2}$ and $\mathrm{Kr}^{+}{ }^{2} P_{1 / 2}$ generates electrons which carry the remainder of the energy, i.e. low-energy electrons are ejected near the respective thresholds only. The electrons ejected as a consequence of an autoionisation process carry energy corresponding to the finite energy difference between this neutral level and the lower lying ionisation threshold. Therefore the coincidence method is rather insensitive to autoionising states if the resolution of the energy analyser is sufficiently high. 


\section{Results and Discussion}

The method has been applied to a number of systems of homogeneous clusters. Illustrative examples are the mass spectra of $\mathrm{Xe}_{n}^{+}$taken at different photon energies, which are shown in Fig. 5. Spectra produced with high-energy photons are very similar to those generated by electron bombardment ionisation, showing prominent mass peaks at $\mathrm{Xe}_{13}^{+}, \mathrm{Xe}_{19}^{+}$and $\mathrm{Xe}_{25}^{+}$[7]. For a given photon energy there is a minimum cluster size for which photoionisation can occur. The clusters disappear altogether below a photon energy of $10.60 \mathrm{eV}$. It has to be noted, however, that the present experimental setup is not sensitive to clusters larger than $n=50$ for reasons of detector sensitivity. It is therefore not surprising that the ionisation energy of liquid $\mathrm{Xe}(9.87 \mathrm{eV}$ as calculated from data contained in Ref. 8) is not reached.

Ionisation efficiency curves of clusters exhibit similar shapes: a slow rise above the ionisation threshold followed by a steep increase to a maximum onto which resonant structures are superimposed. Above a certain energy - which usually coincides with the appearance of fragment ions - the efficiency decreases again. These features are usually interpreted as indirect and direct ionisation channels. While the latter correspond to direct Franck-Condon transitions into the ionic state, the slowly rising part is thought to be caused by transitions via highly excited neutral states which lie close to the curves of the appropriate ion [9]. This process, which is somewhat similar to associative ionisation [10] is usually not efficient, so explaining the low intensity observed near threshold. The latter corresponds more to an adiabatic transition into a cluster geometry of minimum energy while the direct ionisation process corresponds to a vertical transition.

It is therefore useful to note both the threshold energy for photoionisation and the onset of the direct ionisation, which is shown as a function of cluster size in Table 1. The values represent the difference in binding energy between the neutral and the ionic species. The accuracy of the experimental values is approximately $\pm 0.05 \mathrm{eV}$.

\section{Xe 5 bar}

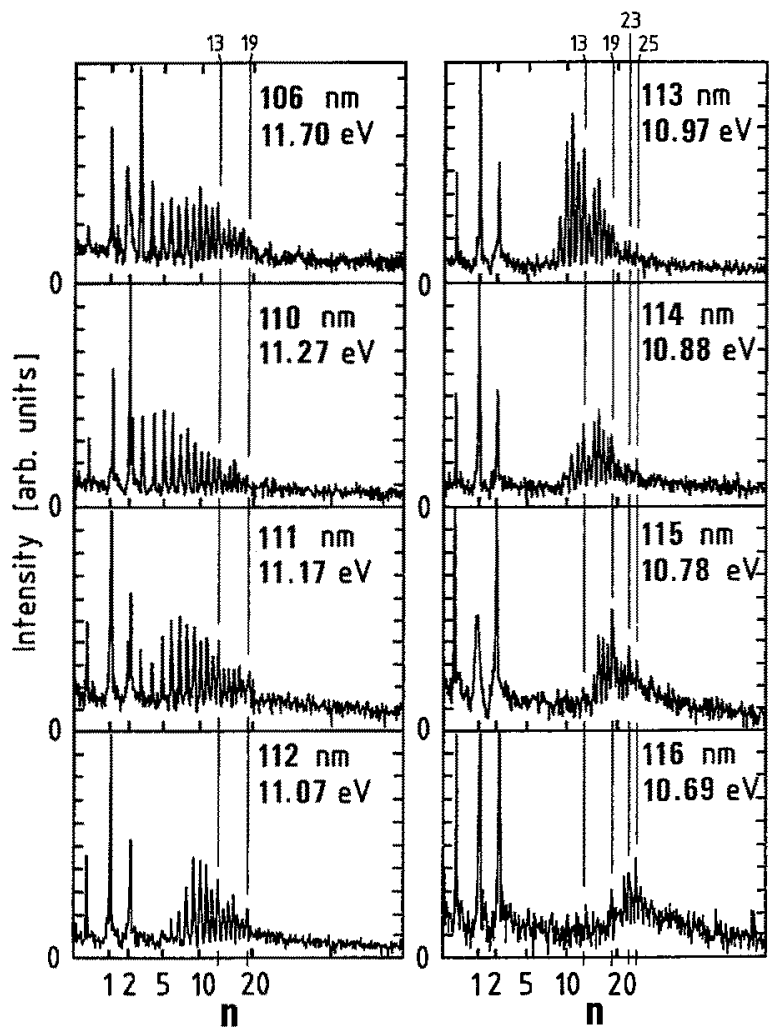

Fig. 5. Photoion-threshold electron coincidence mass spectra of $\mathrm{Xe}$ clusters recorded at different wavelengths. The abscissa shows ciuster size. The monomer peak is due to the finite intensity of the second order of the spectrometer used

The small cluster ions up to $\mathrm{Xe}_{4}^{+}$all appear at the same energy, indicating that the binding energy does not change significantly from the neutral to the ionic cluster. From $n=5$ to $n=13$ the bond energy per particle (i.e. the difference of appearance energy between two successive clusters) is significantly larger, for $n>13$ it decreases again but does not disappear. This is interpreted as due to the shell structure of the ionic cluster [11]: above $n=13$ a second shell is formed with much less binding energy per particle than found for the first shell. From this it is clearly seen that $\mathrm{Xe}_{13}^{+}, \mathrm{Xe}_{19}^{+}$and $\mathrm{Xe}_{25}^{+}$have an appearance energy lower than that of their neighbouring clusters,
Table 1. Photoionisation thresholds of $\mathrm{Xe}_{n}$ clusters in $\mathrm{eV}^{\mathrm{a}}$ (in brackets: onset of the direct ionisation process)

\footnotetext{
a The threshold for $\mathrm{Xe}$ is $12.13 \mathrm{eV}$

${ }^{b}$ No clusters $n<40$ were ionised at $10.60 \mathrm{eV}$
}

\begin{tabular}{|c|c|c|c|c|c|c|}
\hline$n$ & & $n$ & & $n$ & & \\
\hline 2 & $11.00(11.12)$ & 8 & $10.90(10.97)$ & 14 & 10.77 & $(10.82)$ \\
\hline 3 & $11.00(11.12)$ & 9 & $10.85(10.92)$ & 15 & 10.65 & $(10.75)$ \\
\hline 4 & $11.00(11.12)$ & 10 & $10.80(10.85)$ & 16 & 10.65 & $(10.72)$ \\
\hline 5 & $10.95(11.12)$ & 11 & $10.75(10.80)$ & 17,18 & 10.70 & $(10.75)$ \\
\hline 6 & $10.92(11.05)$ & 12 & $10.75(10.80)$ & \multicolumn{3}{|c|}{$19,>23<10.62(<10.65)^{b}$} \\
\hline 7 & $10.92(11.00)$ & 13 & $10.70(10.80)$ & \multicolumn{3}{|c|}{$20,21,22 \quad 10.65$} \\
\hline & & & & liquid $\mathrm{Xe}$ & \multicolumn{2}{|c|}{$9.87[8]$} \\
\hline
\end{tabular}


stressing the exceptionally high stability of these ions. The measured threshold values for the dimer ion are slightly lower than those given by $\mathrm{Ng}$ et al. [6]. Our values for $\mathrm{Xe}_{2}^{+}$and $\mathrm{Xe}_{3}^{+}$are considerably lower than the ones given by Poliakoff et al. [12], which we explain by the higher sensitivity of our experimental setup.

Parallel to the appearance of a lower threshold, the upper limit of the cluster distribution is also found to depend on the photon energy. It is known from experiments with electron bombardment ionisation that large cluster ions are easily formed under the present experimental conditions [13]. This leads us to the conclusion that the surplus energy is transferred to the ejected electron. The particular stability of large cluster ions could be explained by the effect whereby the electron carries away a great fraction of the absorbed energy.

The technical assistance of Mr. J. Lehmann and of the staff of the Berlin synchrotron facility BESSY is gratefully acknowledged.

\section{References}

1. Hutson, J.M., Howard, B.J.: Mol. Phys. 45, 769 (1982); 45, 791 (1982)

2. King, D.L., Dixon, D.A., Herschbach, D.R.: J. Am. Chem. Soc. 963328 (1974)

3. Geusic, M.E., Morse, D.M., Smalley, R.E.: J. Chem. Phys. 82, $590(1984)$

4. Whetton, R.L., Cox, D.M., Trevor, D.J., Kaldor, A.: J. Phys. Chem. 89, $566(1985)$
5. Baer, T.: State selection by photoion photoelectron coincidence. In: Gas phase ion chemistry. Vol. 1. New York: Academic Press 1979; Tanaka, K., Kato, T., Guyon, P.M., Koyano, I.: J. Chem. Phys. 77, 4441 (1982)

Guyon, P.M.: Threshold photoion-photoelectron coincidences. In: Electronic and atomic collisions. Oda, N., Takayanagi, $\mathrm{K}$. (eds). Amsterdam, Oxford, New York: North Holland Publ. Co. 1980

6. Ng, C.Y., Trevor, D.J., Mahan, B.H., Lee, Y.T.: J. Chem. Phys. 66, 446 (1977)

7. Echt, O., Sattler, K., Recknagel, E.: Phys. Rev. Lett. 47, 1121 (1981)

8. Schmidt, W.F.: IEEE Trans. Electron. Ins., EI-19, 389 (1984)

9. Runge, S., Pesnelle, A., Perdrix, M., Watel, G.: Phys. Rev. A 32, 1412 (1985)

10. Carlson, N.W., Taylor, A.J., Schawlow, A.L.: Phys. Rev. Lett. 45, $18(1980)$ Carlson, N.W., Taylor, A.J., Jones, A.J. Schawlow, A.L.: Phys. Rev. A 24, 822 (1981)

11. Saenz, J.J., Soler, J.M., Garcia, N.: Chem. Phys. Lett. 114, 15 (1985)

12. Poliakoff, E.D., Dehmer, P.M., Dehmer, J.L., Stockbauer, R.: J. Chem. Phys, 76, 5214 (1982)

13. Heßlich, J., Ding, A.: Unpublished results

L. Cordis

G. Ganteför

J. Hesslich

A. Ding

Hahn-Meitner-Institut

für Kernforschung Berlin GmbH

Bereich Strahlenchemie

Postfach 390128

D-1000 Berlin 39

Germany 